\title{
Enhanced optical dichroism of graphene nanoribbons
}

\author{
F. Hipolito, ${ }^{1,2}$ A. J. Chaves, ${ }^{3}$ R. M. Ribeiro, ${ }^{3}$ M. I. Vasilevskiy, ${ }^{3}$ Vitor M. Pereira, ${ }^{2, *}$ and N. M. R. Peres ${ }^{3,4, \dagger}$ \\ ${ }^{1}$ NUS Graduate School for Integrated Sciences and Engineering, Centre for Life Sciences, Singapore 117456 \\ ${ }^{2}$ Graphene Research Centre and Department of Physics, National University of Singapore, 2 Science Drive 3, Singapore 117542 \\ ${ }^{3}$ Department of Physics and Centre of Physics, University of Minho, Campus of Gualtar, 4710-057 Braga, Portugal \\ ${ }^{4}$ Academy of Sciences of Lisbon, R. Academia das Ciências 19, 1249-122 Lisboa, Portugal
}

(Received 1 December 2011; published 19 September 2012)

\begin{abstract}
The optical conductivity of graphene nanoribbons is analytical and exactly derived. It is shown that the absence of translational invariance along the transverse direction allows considerable intraband absorption in a narrow frequency window that varies with the ribbon width, and lies in the $\mathrm{THz}$ range domain for ribbons $10-100 \mathrm{~nm}$ wide. In this spectral region the absorption anisotropy can be as high as two orders of magnitude, which renders the medium strongly dichroic, and allows for a very high degree of polarization (up to $\sim 85 \%$ ) with just a single layer of graphene. Using a cavity for impedance enhancement, or a stack of few layer nanoribbons, these values can reach almost $100 \%$. This opens a potential prospect of employing graphene ribbon structures as efficient polarizers in the far IR and THz frequencies.
\end{abstract}

DOI: 10.1103/PhysRevB.86.115430

PACS number(s): 81.05.ue, 72.80.Vp, 78.67.Wj

\section{INTRODUCTION}

Dichroism refers to the ability of some materials to absorb light differently, depending on the polarization state of the incoming wave, and leads to effects such as the rotation of the plane of polarization of light transmitted through them. ${ }^{1}$ This characteristic is the basis of several elementary optical elements like polarizers, wave retarders, etc., which are essential building blocks in optics, photoelectronics and telecommunications. Dichroism, as an intrinsic property of certain materials and substances, is also widely relevant for substance characterization in fields ranging from spectroscopy, to chemistry, to life sciences.

A grid of parallely aligned metallic wires is a well known textbook example of a dichroic system, where unpolarized radiation becomes polarized perpendicularly to the wires, if the wavelength is much larger than the wire separation. ${ }^{2}$ This example shows how geometrical anisotropy can be engineered to induce dichroism in otherwise isotropic media.

Here we unveil the intrinsic dichroic properties of graphene nanoribbons (GNR), and assess how effectively grids of GNRs can be used as polarizing elements. To our knowledge, the intrinsic anisotropic absorption characteristics of GNR have not been explored as we discuss here.

The motivation to explore GNRs in this context comes from a convergence of several critical properties. First, the optical absorption spectrum of pristine graphene is roughly constant over an enormous band of frequencies, ${ }^{3,4}$ from the THz to the near UV. This opens the unprecedented prospect of exploring its optical response to develop optical elements that can operate predictably and consistently in such broad frequency bands. Broadband polarizers, for example, are a much needed element in photonic circuits for telecommunications, and graphene can play here an important role. ${ }^{5}$ Second, the optical absorption of graphene is easily switched on and off by varying the electronic density, which can be easily achieved by electrostatic gating. ${ }^{6}$ Third, due to the record breaking stiffness of the crystal lattice, one can suspend a graphene sheet and cut a grating of the thinnest nanowires (currently of the order of $10 \mathrm{~nm}^{7}$ ), which opens new avenues in ultranarrow gratings, and upon which we base the system depicted in Fig. 1. Fourth, since graphene is metallic and possesses no bulk (it is a pure surface), the rich phenomenology associated with surface plasmons-polaritons (SPP) is certainly unavoidable, further broadening the horizon of possibilities for optical applications. ${ }^{8}$ Finally, the atomic thickness of graphene results in a transparency of $97.7 \%$. Hence, even if one is able to induce strong absorption along one direction, the overall transmissivity will still be large, which is important to maintain losses under control.

\section{DICHROISM MECHANISM}

The natural first step towards such possibilities consists in analyzing the intrinsic optical response of GNRs, to which we dedicate the remainder of this paper. We are interested in how the finite transverse dimension affects the optical absorption spectrum at low frequencies (IR and below), which is rather featureless in bulk graphene ${ }^{3}$ (except for the $\omega=0$ Drude peak), but turns out to be much richer in nanoribbons. The situation we envisage is depicted in Fig. 1, and consists in passing an electromagnetic wave across a grid of GNRs. For definiteness and technical simplicity we restrict our analysis to armchair (AC) nanoribbons, although our results do not depend on the specific chirality, as will be clear later. An important aspect to consider in GNRs has to do with how large edge disorder is expected to be, and to what extent it might mask the phenomena under discussion. To address this, while at the same time keeping as much analytical control over the results as possible, our calculations involve two steps. First, the frequency-dependent conductivity tensor $\sigma_{\alpha \beta}(\omega),(\alpha, \beta=x, y)$ of an AC GNR is derived exactly for free electrons governed by a nearest-neighbor tight-binding Hamiltonian (see below). We then perform ensemble averages of such $\sigma_{\alpha \beta}(\omega)$, where the ribbon width is the fluctuating parameter, and thus extract the overall response of the system accounting for "disorder." This procedure hinges on the assumption that the leading impact of disorder in the optical response is captured by the broadening of the quasi $1 \mathrm{D}$ electronic bands, which is also achieved with an ensemble average of ribbons with 


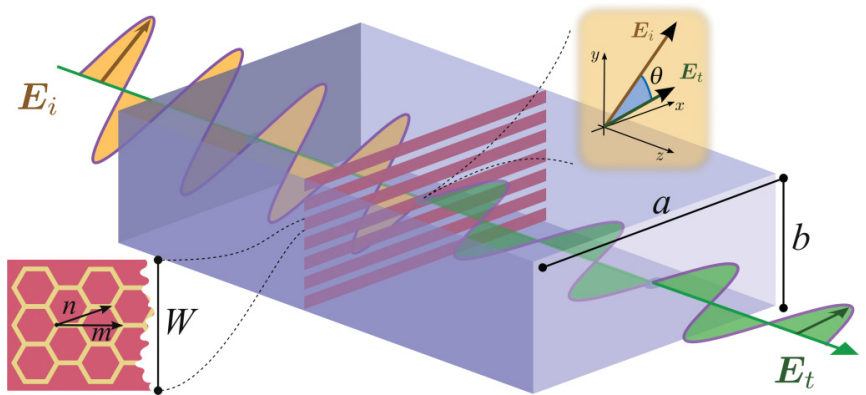

FIG. 1. (Color online) Illustration of the geometry under consideration, and potential device application, consisting of a grid of parallel GNRs perpendicular to the incoming wave. The grid can be in vacuum, at the interface between two different dielectric media $(1,2)$, or even inside a metallic waveguide with sectional area $a \times b$. A plane-polarized incoming wave has its polarization rotated by an angle $\theta$ upon crossing the nanoribbon grating or, alternatively, an unpolarized wave emerges linearly polarized.

fluctuating width. As discussed below, other generic disorder mechanisms (such as carrier density inhomogeneity or strain) are supposed to produce only small (of the order of a few percent) relative fluctuations of the observable properties of the ribbons. Moreover, such ensemble averaging over a distribution of ribbon's widths is also close to the experimental situation, insofar as even state-of-the-art fabrication cannot control ribbon widths with atomic precision. ${ }^{9}$ Thus, an array of ribbons cut out of a graphene sheet will always display a distribution of widths around a predefined target value $\langle W\rangle=W_{0}$. Technically, the conductivity of such an array of GNRs is given by $\left\langle\sigma_{\alpha \beta}(\omega)\right\rangle=\sum_{W} f(W) \sigma_{\alpha \beta}^{W}(\omega)$, where $f(W)$ is the normal distribution for the ribbon width $W$, and $\sigma_{\alpha \alpha}^{W}(\omega)$ is the conductivity of a single ribbon of width $W$.

Overall parametrizations are as follows. The natural energy scale is the hopping amplitude in bulk graphene: $t \simeq 2.7 \mathrm{eV} .^{10}$ The chemical potential $\mu$ determines the free carrier response and also sets the spectral limit for interband transitions (at $T=0 \mathrm{~K}$ ). Nonzero free carrier densities are the norm, and their amount depends on the fabrication and sample treatment procedure. They can range from $n_{e} \sim 10^{10} \mathrm{~cm}^{-2}$ to a few $10^{12} \mathrm{~cm}^{-2}$. Such densities correspond to $\mu$ varying roughly between $0.01 t$ to $0.1 t$, which is the interval we focus on below. Gating allows the carrier density to be easily tuned via field effect. ${ }^{11}$ Ribbons are interchangeably characterized by their absolute width $W$, or by $N$, which counts the number of dimer rows along the transverse direction, and $W=\sqrt{3}(N-$ 1) $a / 2 \simeq 0.12 N \mathrm{~nm}$, where $a \simeq 1.42 \AA$ represents the C-C distance. For the purposes of ensemble averaging, ribbon widths are uniformly distributed with a standard deviation that we take as constant: $\left\langle N^{2}-\langle N\rangle^{2}\right\rangle^{1 / 2}=10(\simeq 1.2 \mathrm{~nm})$. This is done to mimic the experimental limitations associated with the minimum feature size that can be achieved by lithographic means, and is presumably a constant number. All the calculations discussed below have been done for $T=300 \mathrm{~K}$. We use the terms intra- or interband in reference to transitions occurring among subbands with the same or opposite sign of energy, respectively. The hopping amplitude sets the energy scale, and all quantities with dimensions of energy will be expressed in terms of $t$. For $\mu>0.1 t$, and
$N>100(18 \mathrm{~nm})$, the finite width of the ribbon does not significantly alter the relation between $\mu$ and $n_{e}$ from the one in bulk graphene. Hence, $n_{e} \simeq 7 \times 10^{14}(\mu / t)^{2} \mathrm{~cm}^{-2}$. To be definite, for illustration purposes we will take $\mu=0.1 t$ in most of the plots. ${ }^{12}$ Conductivities are normalized to the universal value $\sigma_{0}=\pi e^{2} / 2 h$ of clean $2 \mathrm{D}$ graphene at low frequencies, and the incoming radiation has a wavelength much larger than the ribbon width $W$.

\section{DERIVATION OF THE CONDUCTIVITY TENSOR}

The derivation of the conductivity tensor of an armchair graphene ribbon starts with the consideration of the nearest neighbor tight-binding Hamiltonian describing the $\pi$ bands of graphene, and characterized by a hopping amplitude $t \simeq 2.7 \mathrm{eV} .{ }^{10}$ The ribbon eigenstates have the analytical form ${ }^{13,14}\left|\Psi_{\ell, q, \lambda}\right\rangle=\mathcal{N} \sum_{n, m} e^{-i q(m+n / 2)} \sin \left(k_{\ell} n\right) \times$ $\left(|A, n, m\rangle+\lambda e^{-i \theta_{\ell, q}}|B, n, m\rangle\right)$, where $k_{\ell}=\pi \ell /(N+1)$ is the quantum number associated with transverse quantization $(\ell=$ $1,2, \ldots, N), \mathcal{N}=1 / \sqrt{N+1}, \lambda= \pm 1$ defines the valence $(\lambda=-1)$ or conduction $(\lambda=+1)$ bands, $|A, n, m\rangle$ is the Wannier state at sublattice $A$ of the unit cell at position $\boldsymbol{R}=$ $n \boldsymbol{n}+m \boldsymbol{m}$ (see Fig. 1), $N$ is the number of unit cells along the finite $\boldsymbol{n}$ direction, and $q$ is the dimensionless momentum along $\boldsymbol{m}$, whose value is within the range $-\pi<q \leqslant \pi$. The phase difference between sublattice amplitudes is

$$
\theta_{\ell, q}=\arctan \frac{2 \cos k_{\ell} \sin (q / 2)}{1+2 \cos k_{\ell} \cos (q / 2)} .
$$

This is sufficient to determine the optical conductivity from Kubo's formula: ${ }^{15}$

$$
\begin{aligned}
\sigma_{\alpha \beta}= & \frac{2 i e^{2}}{\omega S} \sum_{\ell_{1}, \ell_{2}, q} \sum_{\lambda_{1}, \lambda_{2}} \frac{f\left(E_{\ell_{1}, q, \lambda_{1}}\right)-f\left(E_{\ell_{2}, q, \lambda_{2}}\right)}{\hbar \omega-\left(E_{k_{2}, q, \lambda_{2}}+E_{k_{1}, q, \lambda_{1}}\right)+i 0^{+}} \\
& \times\left\langle\Psi_{\ell_{1}, q, \lambda_{1}}\left|v_{\alpha}\right| \Psi_{\ell_{2}, q, \lambda_{2}}\right\rangle\left\langle\Psi_{\ell_{2}, q, \lambda_{2}}\left|v_{\beta}\right| \Psi_{\ell_{1}, q, \lambda_{1}}\right\rangle,
\end{aligned}
$$

where $S$ is the area of the ribbon, $f(x)$ the Fermi distribution function, and $\left\langle\Psi_{\ell, q, \lambda}\left|v_{\alpha}\right| \Psi_{\ell^{\prime}, q, \lambda^{\prime}}\right\rangle$ is the matrix element of the $\alpha$ component of the velocity operator. ${ }^{16}$ Since the energy scale is determined by $t$, let us introduce a dimensionless energy parameter $\Omega=\hbar \omega / t$.

Translation invariance along the longitudinal direction dictates that the matrix elements of the velocity $v_{x}$ are diagonal in $q$ and $\ell$, leading to $\sigma_{x x}$ of the form

$$
\Re \frac{\sigma_{x x}}{\sigma_{0}}=\mathcal{N}_{x} \sum_{\ell_{0}} \delta f_{q_{0}, \ell_{0}} M_{x}^{2}\left(q_{0}, \ell_{0}\right),
$$

where $\delta f_{q_{0}, \ell_{0}}=f\left(E_{\ell_{0}, q_{0},-}\right)-f\left(E_{\ell_{0}, q_{0},+}\right), \mathcal{N}_{x}=4 / 3 \sqrt{3}(N-1)$, and $q_{0}$ is given by

$$
q_{0}=2 \arccos \frac{(\Omega / 2)^{2}-1-4 \cos ^{2} k_{\ell_{0}}}{4 \cos k_{\ell_{0}}} .
$$

The sum in Eq. (3) is restricted to those values of $\ell_{0}$ such that $q_{0} \in \mathbb{R}$. Finally, $M_{x}^{2}\left(q_{0}, \ell_{0}\right)$ reads

$$
M_{x}^{2}\left(q_{0}, \ell_{0}\right)=\frac{\left[\cos \theta_{\ell_{0}, q_{0}}-\cos \left(\theta_{\ell_{0}, q_{0}}-q_{0} / 2\right) \cos k_{\ell_{0}}\right]^{2}}{\sin \left(q_{0} / 2\right) \cos k_{\ell_{0}}} .
$$

Only interband transitions (from the subbands with $\lambda=-1$ to $\lambda=+1)$ contribute to $\sigma_{x x}$. 
The analytical expression for $\sigma_{y y}$ is slightly more cumbersome than the previous one, due to the absence of translation invariance along that direction. As a result, (i) the matrix elements of the operator $v_{y}$ are nondiagonal in the subband index $\ell$, and (ii) there are both intraband $\left(\lambda=\lambda^{\prime}\right)$ and interband $\left(\lambda \neq \lambda^{\prime}\right)$ contributions to the transverse conductivity. The calculation is, nevertheless, straightforward, yielding

$$
\mathfrak{R} \frac{\sigma_{y y}}{\sigma_{0}}=\mathcal{N}_{y} \sum_{\ell_{1}, \ell_{2}} \sum_{\lambda, \lambda^{\prime}} \mathcal{P}_{\ell_{1}, \ell_{2}} \delta f_{q_{0}, \ell_{1}, \ell_{2}}^{\lambda, \lambda^{\prime}} M_{y}^{2}\left(q_{0}, \ell_{1}, \ell_{2}\right),
$$

where $\mathcal{N}_{y}=4 / \sqrt{3}(N+1)\left(N^{2}-1\right), \delta f_{q_{0}, \ell_{1}, \ell_{2}}^{\lambda, \lambda^{\prime}}=n_{F}\left(E_{\ell_{1}, q_{0}, \lambda}\right)-$ $n_{F}\left(E_{\ell_{2}, q_{0}, \lambda^{\prime}}\right)$, and $\mathcal{P}_{\ell_{1}, \ell_{2}}=1-(-1)^{\ell_{1}+\ell_{2}}$. This latter factor entails the selection rule for transitions among subbands $\ell_{1}+\ell_{2}=$ odd. The last factor is

$$
\begin{aligned}
M_{y}^{2}\left(q_{0}, \ell_{1}, \ell_{2}\right)= & \frac{\sin ^{2} k_{\ell_{1}} \sin ^{2} k_{\ell_{2}}}{\sin ^{2}\left[\left(k_{\ell_{1}}+k_{\ell_{2}}\right) / 2\right] \sin ^{2}\left[\left(k_{\ell_{1}}-k_{\ell_{2}}\right) / 2\right]} \\
& \times \frac{\epsilon_{\ell_{1}, q_{0}} \epsilon_{\ell_{2}, q_{0}}\left|\sin \left(q_{0} / 2\right)\right|^{-1}(\hbar \omega)^{-1}}{\left|\cos k_{\ell_{1}} \epsilon_{\ell_{2}, q_{0}}+\lambda \lambda^{\prime} \cos k_{\ell_{2}} \epsilon_{\ell_{1}, q_{0}}\right|} \\
& \times \mathcal{C}_{q_{0}, \ell_{1}, \ell_{2}},
\end{aligned}
$$

where $\mathcal{C}_{q_{0}, \ell_{1}, \ell_{2}}=1+\lambda \lambda^{\prime} \cos \left(\theta_{\ell_{1}, q_{0}}+\theta_{\ell_{2}, q_{0}}-q_{0}\right)$, and

$$
q_{0}=2 \arccos \frac{\left(a_{2}-a_{1}\right) Q_{b}+\Omega^{2}\left(b_{1}+b_{2}\right) \pm Q_{c}}{\left(b_{1}-b_{2}\right)^{2}},
$$

with $Q_{c}=2 \sqrt{\Omega^{4} b_{1} b_{2}+\Omega^{2} Q_{b} Q_{a}}, \quad Q_{b}=b_{1}-b_{2}, \quad Q_{a}=$ $b_{1} a_{2}-b_{2} a_{1}, a_{i}=1+4 \cos ^{2} k_{\ell_{i}}$, and $b_{i}=4 \cos k_{\ell_{i}}$. The sum in Eq. (6) is also restricted to those $\ell_{1}, \ell_{2}$ such that $q_{0} \in \mathbb{R}$, and to $\lambda \leqslant \lambda^{\prime}$ (photon absorption only).

The expressions in Eqs. (3) and (6) are our central result, and from them follow all the averages and other physical quantities described and analyzed below.

\section{ANISOTROPIC OPTICAL ABSORPTION}

Lateral confinement reduces the energy spectrum of GNRs to a set of subbands, each reflecting the dispersion of an effective $1 \mathrm{D}$ mode $\ell(\ell=1,2, \ldots, N)$, propagating longitudinally with momentum $q: E_{\ell, q, \lambda}=\lambda t \epsilon_{\ell, q}$, where $\lambda= \pm 1$, defines the valence and conduction subbands,

$$
\epsilon_{\ell, q}=\sqrt{1+4 \cos k_{\ell} \cos (q / 2)+4 \cos ^{2} k_{\ell}},
$$

and $k_{\ell}$ is transverse quantized momentum: $k_{\ell}=\pi \ell /(N+1)$. Consequently, the density of states is dominated by Van Hove singularities (VHS) that develop at $q=0$ for each subband. ${ }^{13,17,18}$ Such sharp spectral features translate into strong optical absorption for ideal GNRs, but are readily smoothed out by edge or bulk disorder and/or temperature in real systems. ${ }^{19}$ Our ensemble averaging has the same effect.

In Fig. 2 we show the averages $\left\langle\sigma_{x x}\right\rangle$ and $\left\langle\sigma_{y y}\right\rangle$ for an ensemble with $\langle N\rangle=150$, and finite chemical potential: $\mu=0.1$. This particular value of chemical potential was chosen to allow a clear distinction between the interband and intraband contributions to the conductivity, so as to better illustrate the main features of the absorption spectrum. As a consequence of time reversal symmetry, only the diagonal components of $\sigma_{\alpha \beta}$ in the coordinate system of Fig. 1 are nonzero. Translation invariance along the longitudinal $(x)$ direction implies that only interband transitions contribute to

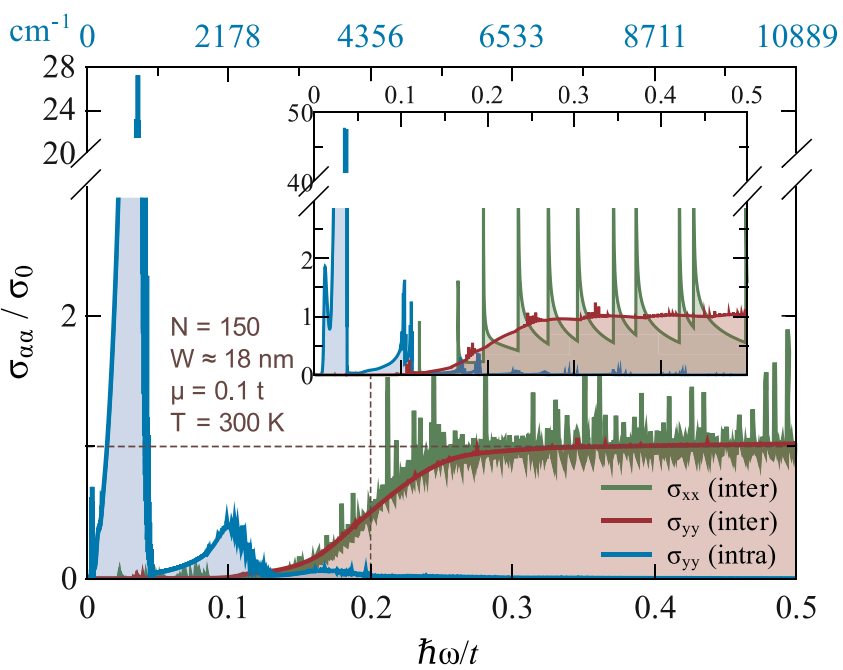

FIG. 2. (Color online) The three nonzero contributions for $\left\langle\sigma_{\alpha \alpha}(\omega)\right\rangle$ discussed in the text, showing a very strong anisotropy in the infrared. In this example the optical conductivities are calculated for an ensemble of ribbons having $\langle N\rangle=150(\simeq 18.5 \mathrm{~nm})$, $\sqrt{\left\langle N^{2}-\langle N\rangle^{2}\right\rangle}=10(\simeq 1.2 \mathrm{~nm})$. We further used $T=300 \mathrm{~K}$ and $\mu=0.1(\simeq 0.3 \mathrm{eV})$. The interband contributions essentially follow the bulk $2 \mathrm{D}$ behavior, with the expected temperature-broadened step onset at $\hbar \omega=2 \mu$. In contrast, the intraband contribution for the transverse conductivity $\left(\sigma_{y y}\right)$ is strongly peaked at low energies. Also note that the vertical axis is truncated for clarity, and that $\sigma_{y y}$ peaks at nearly $28 \sigma_{0}$ for this ensemble. The inset shows the same three quantities, but for a single ribbon of $N=150$, rather than the ensemble.

$\sigma_{x x}(\omega)$, as derived explicitly above. Consequently, $\left\langle\sigma_{x x}(\omega)\right\rangle$ reproduces the bulk 2D behavior, as is clearly seen in Fig. 2. For the analysis of the transverse conductivity, $\left\langle\sigma_{y y}(\omega)\right\rangle$, it is convenient to isolate the inter- and intraband contributions: $\left\langle\sigma_{y y}(\omega)\right\rangle=\left\langle\sigma_{y y}^{\text {inter }}(\omega)\right\rangle+\left\langle\sigma_{y y}^{\text {intra }}(\omega)\right\rangle$ (the latter is allowed since along the transverse direction the electron scatters off the ribbon edges). Whereas $\left\langle\sigma_{y y}^{\text {inter }}\right\rangle$ featurelessly follows $\left\langle\sigma_{x x}\right\rangle$ (and hence the bulk 2D behavior), its intraband counterpart displays a rather strong feature at low energies which, for this specific example, nearly reaches 30 times the universal value $\sigma_{0}$.

Some aspects of Fig. 2 are worth underlying. Firstly, it is evident that, despite averaging to the same step-wise $\omega$-dependence, $\left\langle\sigma_{y y}^{\text {inter }}(\omega)\right\rangle$ is much smoother than $\left\langle\sigma_{x x}^{\text {inter }}(\omega)\right\rangle$, even though the averages are over the same ensemble. This can be traced to the fact that, for each individual ribbon, only $N$ symmetric transitions $(-E \rightarrow+E)$ contribute to $\sigma_{x x}^{\text {inter }}(\omega)$, whereas $\sigma_{y y}^{\text {inter }}(\omega)$ includes $\mathcal{O}\left(N^{2}\right)$ transitions among almost all pairs of subbands. Consequently, the latter has many more absorption singularities, but much weaker, by conservation of spectral weight (this is explicitly shown in the inset of Fig. 2). The averaging is thus more efficient in washing out the structure of VHSs in $\left\langle\sigma_{y y}^{\text {inter }}(\omega)\right\rangle$. Secondly, the low-energy peak in $\left\langle\sigma_{y y}^{\text {intra }}(\omega)\right\rangle$ can be already identified from a single ribbon (inset). Its origin is simple to understand with reference to Fig. 3. Since the band structure consists of a set of discrete subbands, the chemical potential will always be straddled by two of them at $q=0$, such that 

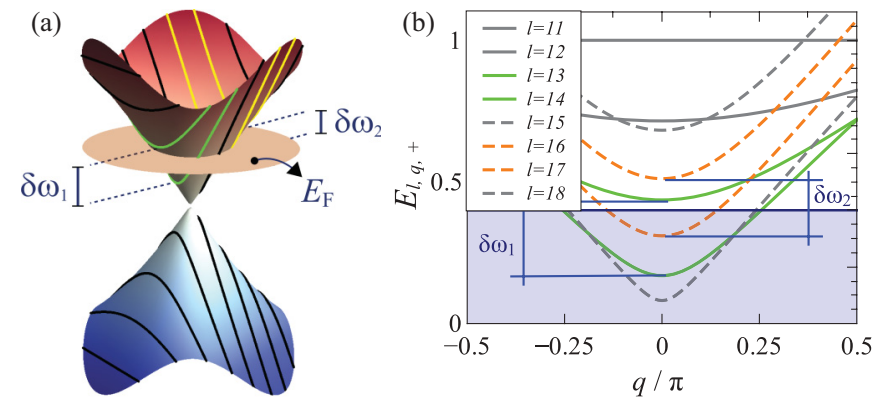

FIG. 3. (Color online) (a) Illustration of the transverse spectrum quantization and highlight of the two most significant intraband transitions $\delta \omega_{1,2}$, which appear around the Dirac point $(N=21$, $E_{F}=0.4 t$ ). (b) The projection of the subbands $E_{\ell, q,+}$ shown in (a), as a function of the 1D momentum $q$, and for $l=11, \ldots, 18$ (see text).

$E_{\ell, q=0, \lambda}<\mu<E_{\ell+1, q=0, \lambda}$. Given that transitions $\ell \rightarrow \ell+1$ are allowed in $\sigma_{y y}^{\text {intra }}$, one expects an absorption peak at $\hbar \omega \approx$ $\left|E_{\ell, q, \lambda}-E_{\ell+1, q, \lambda}\right|$. Moreover, as per Eq. (7) the matrix element decays rapidly with the difference in band index, so that the transitions between the two bands closest to $\mu$ completely dominate $\sigma_{y y}^{\text {intra }}$. From Fig. 3 it is clear that there are always two pairs of such bands, whose energy difference at $q=0$ is $\hbar \delta \omega_{1,2} \approx \pi \sqrt{3-\mu^{2} \pm 2 \mu} /(N+1)$. Since we are interested in situations where $\mu \ll 1$, the intraband peaks are solely determined by the ribbon geometry: $\hbar \omega_{\max } \approx \pi \sqrt{3} /(N+1)$. This can be confirmed in the inset of Fig. 5 for ensembles with different $\langle N\rangle$, and introduces an element of predictability and tunability with respect to the frequency band where the optical absorption is highly enhanced. In other words, given the frequency of operation desired for a given application, one can select the appropriate average ribbon width that yields the strongest optical anisotropy at that target frequency.

Another relevant detail to notice is that, as seen in Fig. 2, the absorption peak in $\sigma_{y y}^{\text {intra }}$ is much more resilient to the ensemble averaging (or level broadening) than all the other transitions coming from interband processes: the averaging readily washes out the VHS features, but leaves the peak in $\left\langle\sigma_{y y}^{\text {intra }}\right\rangle$ quite well defined and intense. In the case shown in Fig. 2, $\left\langle\sigma_{y y}^{\text {intra }}\right\rangle$ peaks at a few dozen times the value of the longitudinal $\left\langle\sigma_{x x}\right\rangle$ The reason for this is very simple to understand qualitatively, and is twofold. On the one hand, since there are always two resonant conditions very close in frequency (for example, $\delta \omega_{1}$ and $\delta \omega_{2}$ in Fig. 3), the shape of the feature in $\sigma_{y y}^{\text {intra }}$ has a double peak structure. To show this explicitly, in Fig. 4 we present a close-up of $\sigma_{y y}^{\text {intra }}$ for the single ribbon with $N=150$ previously shown in the inset of Fig. 2: the double peak structure is self-evident. In addition to that, the transition processes contributing to $\sigma_{y y}^{\text {intra }}$ are quite different from the ones that contribute to $\sigma_{x x}$, or $\sigma_{y y}^{\text {inter }}$. In a single independent ribbon, the longitudinal conductivity is dominated by interband transitions among subbands which have an inverted dispersion with respect to each other [see Fig. 4(b) for an illustration]. Consequently the resonant condition occurs only at the van Hove point, leading to the very sharp van Hove absorption peaks in $\sigma_{x x}$ that we see in the inset of Fig. 2. In contrast, the processes contributing the most to $\sigma_{y y}^{\text {intra }}$ involve transitions among nearly parallel subbands [Fig. 4(b)], thus
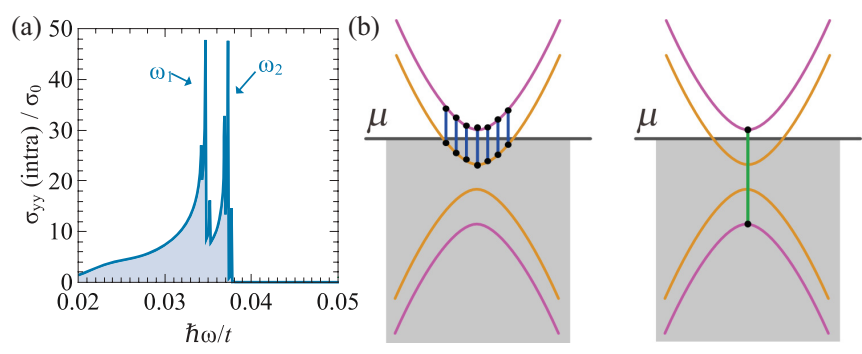

FIG. 4. (Color online) (a) A close-up of the most prominent feature in $\sigma_{y y}^{\text {intra }}$ for the single ribbon whose absorption spectrum was shown in the inset of Fig. 2. On this close-up the double peak structure discussed in the text is clearly visible. (b) An illustration of the different nature of the processes contributing to $\sigma_{y y}^{\text {intra }}$ and $\sigma_{x x}^{\text {inter }}$. In the former, the resonant transitions occur between quasi-parallel subbands, whereas in the latter the resonant condition is only strictly verified at the van Hove point.

allowing a finite density of momentum states to contribute to the resonance, and implying a larger joint density of states. This makes the absorption feature in $\sigma_{y y}^{\text {intra }}$ broader than the van Hove-type peaks associated with $\sigma_{x x}$. The consequence of this is that, when one considers the ensemble averaging, the sharp van Hove peaks in the longitudinal conductivity will be slightly displaced with the changing $N$ within the ensemble, and are rapidly washed out. The double-peak structure, combined with the broader parallel-dominated absorption, protects the transverse absorption peak with respect to the level broadening, thereby resulting in an absorption feature that is much more robust.

To assess the polarizing efficiency of a single graphene ribbon we calculate the optical transmission amplitude, which is the ratio of the electric field amplitudes of the incoming and transmitted fields: $t_{\alpha}(\omega)=E_{\alpha}^{(t)} / E_{\alpha}^{(i)},(\alpha=x, y)$. For radiation impinging normally upon an ensemble of GNRs separating medium 1 and medium 2 (Fig. 1), the transmission amplitude reads explicitly

$$
t_{\alpha}(\omega)=\frac{2 Z^{(2)}}{Z^{(1)}+Z^{(2)}\left[1+Z^{(1)}\left\langle\sigma_{\alpha \alpha}(\omega)\right\rangle\right]},
$$

where $Z=\sqrt{\mu_{0} \mu / \epsilon_{0} \epsilon}$ is the impedance of each medium. This result is obtained in the conventional way, by assuming that the system of graphene ribbons is a metallic sheet of zero thickness, and imposing the boundary conditions of the electromagnetic field at the interface. Knowledge of $t_{\alpha}(\omega)$ allows for the calculation of the degree of polarization [DP, $\mathcal{P}(\omega)]$, or the rotation of the plane of linear polarization $\left(\theta=\theta_{f}-\theta_{i}\right)$ :

$$
\mathcal{P}(\omega)=\frac{\left|t_{x}\right|^{2}-\left|t_{y}\right|^{2}}{\left|t_{x}\right|^{2}+\left|t_{y}\right|^{2}}, \quad \tan \theta_{f}=\frac{t_{y}(\omega)}{t_{x}(\omega)} \tan \theta_{i},
$$

This definition is useful for unpolarized incoming light where $\mathcal{P}= \pm 1$ reflects full polarization of the incoming wave. For an already polarized incoming wave, the second equation shows that the effect naturally depends on the orientation of the incoming polarization with respect to the ribbon principal directions. With $\mathcal{P}(\omega)$ we can immediately identify the degree of dichroism by how close $|\mathcal{P}(\omega)|$ is to unity (i.e., how close to an ideal polarizer we are). 


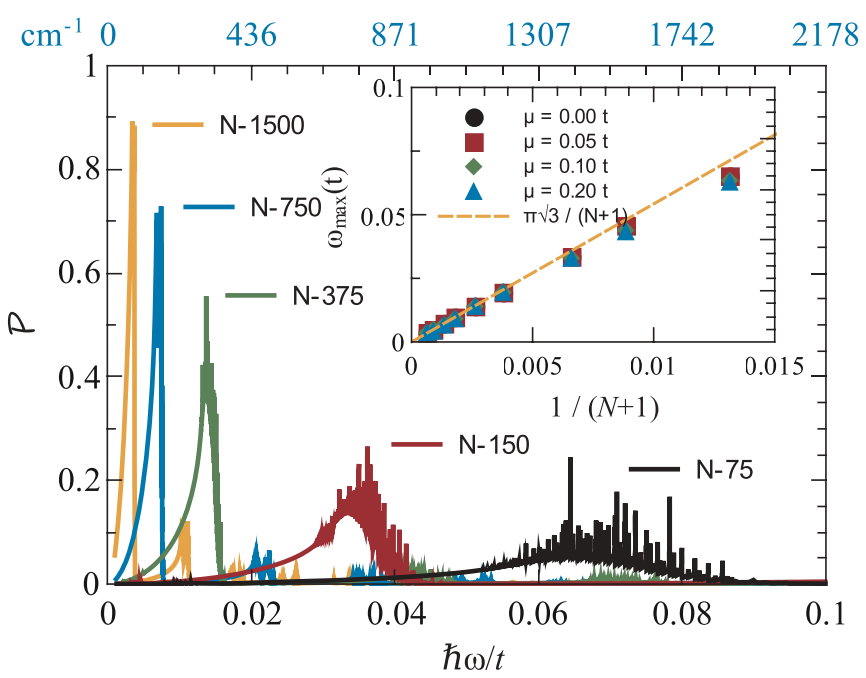

FIG. 5. (Color online) The degree of polarization $\mathcal{P}(\omega)$ in the low energy region, for ribbons of different average width (in unit cells) $\langle N\rangle$ and $\mu=0.1, T=300 K$ (for reference, $\langle N\rangle=\{75,150$, $375,750,1500\} \Leftrightarrow\langle W\rangle=\{9,18,46,92,184\} \mathrm{nm})$. The inset shows the position of the most prominent peak in $\left\langle\sigma_{y y}^{\text {intra }}\right\rangle$ as a function of $\langle N\rangle$ and $\mu$. The $\mu$ dependence is expectedly weak, while the peak position is seen to follow the analytical form described in the text.

In Fig. 5 we plot $\mathcal{P}(\omega)$ for different ribbon widths. It can be clearly seen that DP in excess of $50 \%$ can be achieved already with ribbons $45 \mathrm{~nm}$ wide. We underline that this is the degree of polarization produced by an atomically thin ensemble of ribbons, which makes the magnitude of the effect even more striking! Even though the transparency of infinite 2D graphene is as large as $97.7 \%$, the confinement-induced anisotropy can be so large as to almost completely suppressing one of the field projections. The same figure also confirms that the optimum DP is achieved at a width-dependent frequency $\omega_{\max }$ which, as discussed above, has a simple form (inset of Fig. 5). However it is also clear that this tunability is at the expense of the absolute amount of DP [narrower ribbons $\rightarrow$ larger $\omega_{\max } \rightarrow$ smaller $\left.\mathcal{P}\left(\omega_{\max }\right)\right]$. Nevertheless, it has been experimentally confirmed that the optical absorption of $N$-layer graphene is simply proportional to $N$, from the bilayer to graphite ${ }^{20}$ for most of the low energy range. ${ }^{3,21,22}$ This means that the effect reported here can be significantly magnified by using few-layer graphene ribbons, or simply superimposing a few independent layers onto each other.

In addition, the form of Eq. (10) given in terms of the impedance of the media suggests that additional parameter freedom can be achieved if the wave propagates inside a metallic waveguide. As is well known, electromagnetic propagation in waveguides is restricted to normal TEM, TM, or TE modes. Each of the latter two has a characteristic dispersion that is different from the free-space relation $\omega=$ $c k / n$. For the purpose of analyzing transmission and reflection amplitudes in a situation as depicted in Fig. 1, the effect of the waveguide can be absorbed in a renormalized and frequencydependent impedance $Z(\omega)$. For example, the mode $\mathrm{TE}_{m n}$ has a characteristic impedance ${ }^{23} Z_{m n}(\omega)=Z \omega / \sqrt{\omega^{2}-\omega_{m n}^{2}}$, where $\omega_{m n}^{2}=\left(c^{2} \pi^{2} / \mu \epsilon\right)\left[(m / a)^{2}+(n / b)^{2}\right]$. Hence each mode can only propagate if $\omega$ is beyond the mode cut-off frequency $\omega_{m n}$, and this is frequently used to select/restrict the propagating

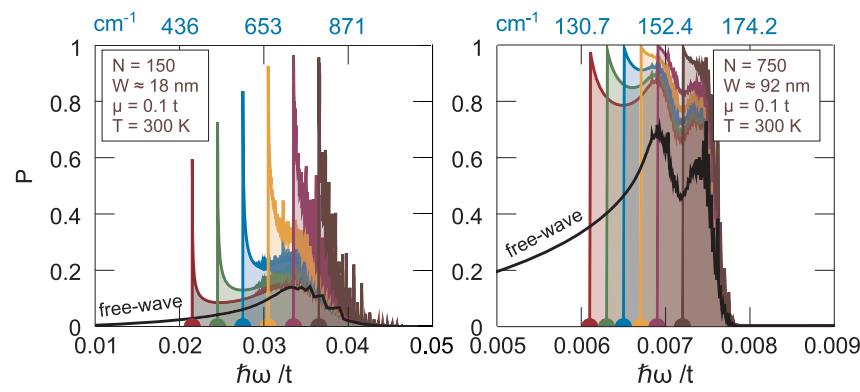

FIG. 6. (Color online) The effect of a metallic waveguide of square cross section in the degree of polarization $\mathcal{P}(\omega)$ for two ensembles of ribbons $(\langle N\rangle=150,750)$. Each panel shows $\mathcal{P}(\omega)$ for an incoming wave made of a combination ${ }^{24}$ of the two lowest degenerate modes $\mathrm{TE}_{10,01}$, in vacuum (black) and in waveguides (colors) with different geometries, i.e., different cutoff frequency $\omega_{10}$. Each $\omega_{10}$ is marked by a dot at the corresponding $w$ in the horizontal axis and a unique color.

modes by adapting the geometry of the waveguide. In our example we could take a square cross section $(a=b)$, in which case the two degenerate modes $\mathrm{TE}_{10}$ and $\mathrm{TE}_{01}$ can be combined into an arbitrary incoming plane polarization. ${ }^{24}$ In that case, if $\omega_{10}<\omega<\omega_{11}$, only the modes TE $E_{10,01}$ propagate in the waveguide, and $Z_{10}(\omega)=Z \omega / \sqrt{\omega^{2}-\omega_{10}^{2}}$. The cavity setup is interesting and useful for two reasons, which can be understood by inspection of Fig. 6: (i) on one hand, by tuning the cavity dimensions so that $\omega_{10} \lesssim \omega_{\max }(N)$ one can precisely cut off the DP below $\omega_{10}$, creating a well defined band of frequencies where the system displays high DP; (ii) on the other hand, since $Z_{10}(\omega)>Z$ (and, in particular $\left.Z_{10}\left(\omega \gtrsim \omega_{10}\right) \gg Z\right)$, the cavity highly magnifies the DP, even for a monolayer system. Taking as illustration the ribbon ensemble with $\langle N\rangle=750$ shown in Fig. 6, proper tuning of the cutoff frequency can introduce a clear and well defined band filter for $\mathcal{P}(\omega)$, while simultaneously amplifying the magnitude of $\mathcal{P}(\omega)$ in comparison with the value for a free wave. $[\mathcal{P}(\omega)$ climbs beyond $80 \%$ in the entire frequency window]. Lastly, this enhancement of the impedance can also make $\mathcal{P}(\omega)$ more steplike within the strongly amplified regime, rather than peaklike, as implied by the right panel of Fig. 6.

\section{DISCUSSION}

The optical absorption of a ribbon is seen here to be highly anisotropic on account of the new intraband channel made possible by the finite transverse direction, and the resulting electron scattering at the ribbon edges. Recent experiments do show that the transmission spectrum of graphene ribbon arrays is rather different for light polarized parallel and perpendicularly to the ribbon length, with the latter dominated by a plasmon absorption resonance at $\sim 3 \mathrm{THz} .{ }^{8}$ However, these experiments pertain to ribbons much wider ( $\gtrsim 1 \mu \mathrm{m})$ than the ones envisaged here $(\lesssim 50 \mathrm{~nm})$, such that their spectrum is effectively continuous. Naturally, in the limit of wide ribbons $(N \rightarrow \infty)$, the peak of $\sigma_{y y}$ in Fig. 2 simultaneously narrows and moves towards $\omega=0$, where it becomes the Drude singularity that we expect for an infinite and disorder-free system. Indeed, the easiest way to understand the sharp feature 
of $\sigma_{y y}$ at low energies is to see it as a usual Drude peak that has been shifted to finite $\omega$ by making the system finite along the transverse direction, thus allowing intraband transitions of finite frequency.

The issue of how to actually manufacture a grid of narrow GNRs with consistent and predictable width has been addressed earlier. It can be achieved by means of high precision patterning using a He-ion beam microscope in lithography mode, ${ }^{7}$ or more standard etch masks able to cut down to the $10 \mathrm{~nm}$ scale. ${ }^{25}$ An alternative to cutting ribbons out of graphene sheets is the recently developed technique of unzipping carbon nanotubes (CNTs). ${ }^{26-28}$ Nowadays it is possible to produce batches of CNTs with similar radius, ${ }^{29}$ and so this would allow for the production of high quality ribbons without edge disorder. Another alternative, that completely bypasses patterning, consists in inducing effective nanoribbons by engineering a periodic distribution of strain in a bulk graphene sheet, such that the strain-induced confinement mimics the ribbon quantization features. ${ }^{30}$

As always in the context of GNRs, the role of disorder needs to be addressed, and perhaps electron-electron interactions as well. ${ }^{15}$ It is known that disorder can affect and even destroy many intrinsic features, such as the edge modes in zig-zag (ZZ) GNRs ${ }^{17}$, the spontaneous spin polarization expected for ideal $\mathrm{ZZ}$ ribbons, ${ }^{18,31}$ the width scaling of the gap, ${ }^{32,33}$ or their conductance. ${ }^{34}$ In our case, disorder can modify the intrinsic optical anisotropy in different ways, depending on the causes: (i) inhomogeneities of the free carrier density caused by various external effects (e.g., substrate inhomogeneities, adsorbates, charged impurities); (ii) spatial fluctuations of the site energy and hopping parameters leading to broadening of mini-bands and carrier scattering, which in turn broadens and shifts the intraband absorption peaks; (iii) adsorbates and other impurities can introduce spurious features in the absorption spectrum; (iv) edge disorder can lead to localization of some electronic states. ${ }^{34}$ Concerning (i), typical electron density fluctuations in graphene on representative substrates, such as $\mathrm{SiO}_{2}$, have been evaluated experimentally, ${ }^{35}$ and seen to be of the order of $\delta n_{e} \sim 4 \times 10^{10} \mathrm{~cm}^{-2}$ in relatively clean systems. Such effects will presumably have little impact when the overall carrier density is between $10^{11}$ and $10^{12}$, which are the densities targeted in our study. The effects of diagonal and nondiagonal disorder (ii) are expected to be less important for narrower ribbons, simply because the anisotropy is induced by intra subband absorption, and the separation of the subbands scales as $\propto 1 / N$ (and so the narrower the ribbon the less significant become local fluctuations of the potential energy, or the hopping amplitudes). Therefore, it is expected that the necessary anisotropy in $\sigma(\omega)$ might be achieved in practice. Regarding (iii), post-patterning annealing techniques have been progressively improved, and proven quite efficient in removing such sources of disorder; ${ }^{36}$ alternatively, encapsulation of graphene has been shown to significantly reduce environmental contamination and to reduce electronic scattering. ${ }^{37}$ With respect to (iv), much depends on the fabrication technique, and the CNT unzipping method (or perhaps the strain-engineering route) would be preferred to mitigate edge disorder. If present in a strong degree, however, edge disorder might bring about new effects not considered here. In particular, experiments show that edge disorder arising from conventional lithographic procedures leads to strong electron localization, and the emergence of a system of effective coupled quantum dots, where charging and interaction effects can be important. ${ }^{32,38}$ The extent to which these features modify the absorption spectrum is not known experimentally and, theoretically, a realistic approach to the problem is out of range of a fully analytical approach, as we seek and use here. These effects will be addressed in future work.

Another issue to consider is the low frequency absorption characteristic of any metal, associated with disorder-induced intraband transitions, and accounted for by the Drude model. In the case of graphene, the Drude conductivity is given by

$$
\frac{\sigma_{D}}{\sigma_{0}}=\frac{4|\mu|}{\pi} \frac{1}{\hbar(\gamma-i \omega)}
$$

where $\gamma$ is the Drude scattering rate. For nanoribbons, such a term would have to be added to $\sigma_{x x}$. The appearance of a Drude peak at $\omega=0$ is not expected to drastically affect the absorption peaks discussed so far, which occur at $\omega=\omega_{\max }$ (finite). A similar conclusion was drawn in recent experiments measuring optical absorption in nanoribbons much wider than our target widths (and so quantization effects disappear there), which show anisotropic absorption features dominated by plasmon absorption, which are vastly insensitive to the Drude component. $^{8}$

At any rate, to be more quantitative, the typical Drude scattering rate lies in the vicinity of $100 \mathrm{~cm}^{-1}(=0.005 t) .^{39}$ Thus, in view of the results of Fig. 5, the Drude regime should only dominate for ribbons of average width above $184 \mathrm{~nm}(\langle N\rangle \gtrsim 1500)$. Such ribbons are too wide anyway for the sort of dimensions we are primarily interested in, which lie around $50 \mathrm{~nm}$ or below $(\langle N\rangle \lesssim 375)$, and for which we find DP in excess of $50 \%$ already. In addition, Fig. 2 shows that the magnitude of the peak in the transverse conductivity easily reaches 10-20 times the value $\sigma_{0}$. For wider ribbons than the one shown $(184 \mathrm{~nm})$ the peak easily surpasses a factor of 100 , even after an ensemble average has been performed (see, e.g., Fig. 7).

The Drude peak, on the other hand, has a magnitude given by $\Re\left[\sigma_{D}(\omega=0) / \sigma_{0}\right]=4|\mu| /(\hbar \gamma) \approx 800|\mu| / t$. For $\mu=0.1 t$ this means that $\Re\left[\sigma_{D}(\omega=0) / \sigma_{0}\right] \approx 80$. However, if we lower the Fermi energy by a factor of 10 to $\mu=0.01 t$, its magnitude will be 10 times smaller, of course, but the change in the transverse absorption peak is not so significant. An example of this is shown in Fig. 7, where we show the effect of decreasing $\mu$ (i.e., lowering the carrier density), both on the degree of polarization, and on $\left\langle\sigma_{y y}^{\text {intra }}(\omega)\right\rangle$. At $\mu=0.01 t$ ( $n_{e} \simeq 7 \times 10^{10} \mathrm{~cm}^{-2}$ ) both the polarizability and the transverse conductivity peak remain significant. In other words, one can suppress the amplitude of the Drude peak at lower densities while not suppressing much the anisotropy and polarizability.

As we pointed out already, by considering the response of an ensemble of GNRs with fluctuating widths, we are introducing considerable broadening effects already [compare, for example, the peak in $\left\langle\sigma_{y y}(\omega)\right\rangle$ for an ensemble in Fig. 2, with the five times more intense peak of a single ribbon (inset)]. For these reasons, we believe that the dichroism of GNRs remains considerably enhanced in the presence of 

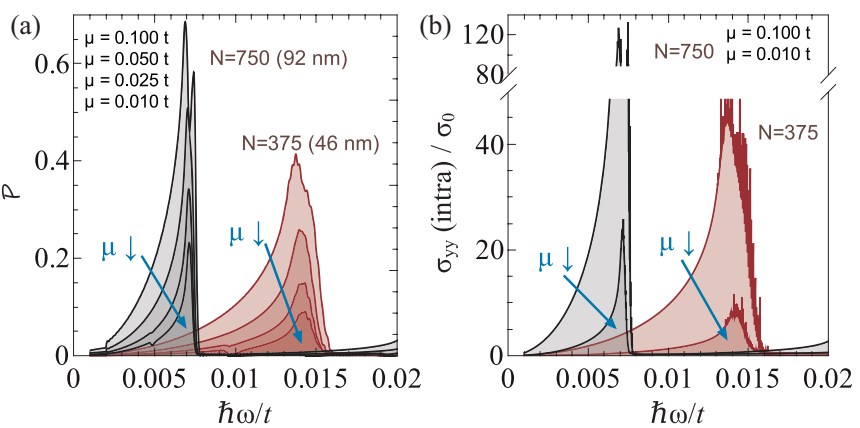

FIG. 7. (Color online) (a) The degree of polarization $\mathcal{P}(\omega)$ as a function of Fermi energy. The behavior at low energies is shown for two ensembles of ribbons of average width $\langle N\rangle=750(92 \mathrm{~nm})$ and $\langle N\rangle=375(46 \mathrm{~nm})$ and, for each, the Fermi energy is varied from $\mu=0.01 t$ to $\mu=0.1 t$. Lowering $\mu$ leads to the progressive decrease in the magnitude of the degree of polarization. (b) The same analysis but now for $\left\langle\sigma_{y y}^{\text {intra }}(\omega)\right\rangle$, and considering only the two extreme values of $\mu$. Notice how the vertical scale is truncated, and that the transverse conductivity in the case $\langle N\rangle=750$ peaks at $120 \sigma_{0}$ for $\mu=0.1 t$, and at $25 \sigma_{0}$ for $\mu=0.01 t$.

realistic moderate disorder. It is worth highlighting also the fact that, since the dichroism stems here from purely spectral considerations, the chirality of the GNRs should be immaterial. In fact, all ribbons have the same scaling of the spectral features with $N$, irrespective of their chirality, and so we expect the dichroism to remain when the ensemble comprises GNRs of arbitrary chirality.

Finally, having in mind the scheme depicted in Fig. 1 where we propose a grating of GNRs, we point out that the dichroism discussed here so far is intrinsic to each element of the grating, as it were. This is a departure from the conventional situation where the grating is made from a normal (isotropic) metal, and the polarizing effect arises from the geometry only, not from some intrinsic anisotropy of the metallic comb itself. In fact, it might have been noted that, whereas a conventional metallic grating polarizes perpendicularly to the slit direction, the dichroism of the individual GNRs favors polarization along the ribbon direction. The actual overall polarizing characteristics of a periodic grating based on GNRs would have to be determined by the combination of this intrinsic dichroism with the geometrical effect (just as in a conventional grating), and for which the surface plasmon-polariton (SPP) physics may play an important role. ${ }^{40}$ However, SPP excitations contribute to the optical absorption only if: (i) the incoming wave's frequency coincides with the band where those excitations are allowed, and not damped; (ii) the grating is strictly periodic; (iii) all elements of the grating are metallically connected so as to maintain coherence of the excitations across the system as a whole; (iv) the incoming wave impinges the grating at oblique incidence. Given that we consider only normal-incidence (which is the one typically most straightforward and efficient from an experimental/applications point of view), the last condition (iv) is violated from the outset, and corrections to the DP arising from SPP are not expected. Moreover, one crucial reason for the existence frequency bands of strong SPP absorption (or transmission) in 3D metallic gratings arises from the coupling between those modes at the two opposing surfaces. ${ }^{40}$ Being a strict 2D metallic system (in effect a metallic boundary condition for the propagation of electromagnetic waves), SPP cannot decay into the (nonexistent) bulk of graphene. This points to the peculiarities of the SPP physics in this 2D Dirac metal, which have been addressed in detail in Ref. 41 . In particular, this reference identifies the conditions for the existence of SPP modes, concluding that they are only allowed in a the range of frequencies close to the DC limit, where the optical response is dominated by the Drude peak. Hence, with respect to point (i) above, even if one considers the possibility of oblique incidence, the conditions for excitation of SPP are rather narrow, and not expected to play a role at the finite frequencies where the DP effect of the ribbon system is most effective (see more below). Points (ii) and (iii) strongly depend on the fabrication process leading to the ribbons and/or their integration in the final gratings, and are easily controllable. The main message we wish to underline in this context is then that, effects associated with increased absorption within certain frequency bands arising from SPP are not expected in the context of our proposed setup, and will not influence the DP. But they could as well be explored by enforcing the conditions enumerated above, and possibly allow even more versatility and richness to the polarizing characteristics of nanoribbon-based gratings. Such considerations are, however, out of the scope of this report.

\section{CONCLUSIONS}

Having derived the exact optical conductivity tensor of GNRs, we studied the optical absorption response of ensembles of ribbons with fluctuating width. One verifies that the optical absorption can be made highly anisotropic within a frequency band that is tunable via the ribbon average width, and/or via the impedance characteristics of the embedding medium. Physically, the origin of such strong anisotropy lies in a resonant feature that is simultaneously very strong and resilient to level broadening, in comparison with the conventional van Hove-type absorption singularities, which quickly wash out in the presence of width fluctuations and/or disorder.

Quantitative analysis reveals that an ensemble of monolayer GNRs can show a very high degree of polarization, $\sim 85 \%$. This value can be enhanced by placing the ribbon in a cavity, so that the real part of the impedance is increased in the appropriate region of the spectrum. In such situations the degree of polarization can be close to $100 \%$, which is quite remarkable given the atomic thickness of the polarizing element.

The current analysis focuses on the intrinsic absorption anisotropy of GNRs, where disorder effects are mimicked by the fluctuating ribbon widths. We are currently exploring routes to study the influence of more specific disorder models, and combining the intrinsic absorption response of GNRs with the geometric effects expected to arise in a GNR grating setup. Likewise, the interplay of the anisotropy induced here by space quantization and plasmons likely to be excited in such finite-sized geometries should be addressed in the future.

Given the recent developments in precision patterning and growth of narrow GNRs, and given the technological interest in optical elements operating in the IR and THz bands, we trust these results can motivate further theoretical and experimental 
investigation of GNRs and other graphene-derived structures towards such applications.

\section{ACKNOWLEDGMENTS}

We acknowledge insightful discussions with A. H. Castro Neto and J. M. B. Lopes dos Santos. F.H. acknowledges partial support from Grant No. UMINHO/BI/ 001/2010. A.J.C., R.M.R., M.I.V., and N.M.R.P. acknowledge support from FEDER-COMPETE, and from FCT Grant No. PEst-C/FIS/UI0607/2011. V.M.P. acknowledges the support of NRF-CRP award "Novel 2D materials with tailored properties: beyond graphene" (R-144-000-295-281). *vpereira@nus.edu.sg

†peres@ fisica.uminho.pt

${ }^{1}$ M. Born and E. Wolf, Principles of Optics (Cambridge University

Press, Cambridge, 1997).

${ }^{2}$ M. H. Fizeau, Ann. Chim. (Phys.) 63, 385 (1861).

${ }^{3}$ R. R. Nair, P. Blake, A. N. Grigorenko, K. S. Novoselov, T. J. Booth, T. Stauber, N. M. R. Peres, and A. K. Geim, Science 320, 1308 (2008).

${ }^{4}$ N. M. R. Peres, Rev. Mod. Phys. 82, 2673 (2010).

${ }^{5}$ Q. Bao, H. Zhang, B. Wang, Z. Ni, C. H. Y. X. Lim, Y. Wang,

D. Y. Tang, and K. P. Loh, Nat. Photon. AOP (2011).

${ }^{6}$ Z. Q. Li, E. A. Henriksen, Z. Jiang, Z. Hao, M. C. Martin, P. Kim, H. L. Stormer, and D. N. Basov, Nat. Phys. 4, 532 (2008).

${ }^{7}$ M. C. Lemme, D. C. Bell, J. R. Williams, L. A. Stern, B. W. H. Baugher, P. Jarillo-Herrero, and C. M. Marcus, ACS Nano 3, 2674 (2009).

${ }^{8}$ L. Ju, B. Geng, J. Horng, C. Girit, M. Martin, Z. Hao, H. A. Bechtel, X. Liang, A. Zettl, Y. R. Shen et al., Nat. Nanotechnol. 6, 630 (2011).

${ }^{9}$ G. Xu, Jr., J. Bai, J. Tang, T. Yu, Y. Huang, X. Duan, Y. Zhang, and K. L. Wang, Appl. Phys. Lett. 98, 243118 (2011).

${ }^{10}$ A. H. C. Neto, F. Guinea, N. M. R. Peres, K. S. Novoselov, and A. K. Geim, Rev. Mod. Phys. 81, 109 (2009).

${ }^{11}$ K. S. Novoselov, A. K. Geim, S. V. Morozov, D. Jiang, Y. Zhang, S. V. Dubonos, I. V. Grigorieva, and A. A. Firsov, Science 306, 666 (2004).

${ }^{12}$ This corresponds to $n_{e} \sim 10^{12} \mathrm{~cm}^{-2}$, which nowadays is routinely obtained, and gate-tunable, by means of ionic or electrolyte enhanced gating (Refs. 8, 42, and 43).

${ }^{13}$ K. Wakabayashi, K.-i. Sasaki, T. Nakanishi, and T. Enoki, Sci. Technol. Adv. Mater. 11, 054504 (2010).

${ }^{14}$ J. Ruseckas, G. Juzeliunas, and I. V. Zozoulenko, Phys. Rev. B 83, 035403 (2011).

${ }^{15}$ N. M. R. Peres, R. M. Ribeiro, and A. H. Castro Neto, Phys. Rev. Lett. 105, 055501 (2010).

${ }^{16}$ T. Stauber, N. M. R. Peres, and A. K. Geim, Phys. Rev. B 78, 085432 (2008).

${ }^{17}$ M. Fujita, K. Wakabayashi, K. Nakada, and K. Kusakabe, J. Phys. Soc. Jpn. 65, 1920 (1996).

${ }^{18}$ K. Wakabayashi, M. Fujita, H. Ajiki, and M. Sigrist, Phys. Rev. B 59, 8271 (1999).

${ }^{19}$ The situation with nanotubes is quite different, because the tubes have no edges, and hence are very weekly affected by disorder. Their intrinsic absorption spectrum and the transitions between subbands are very well reproduced experimentally. But the analogous has not been seen in GNR yet, due to the (so far) unavoidable edge roughness.
${ }^{20}$ Regarding perfectly $\mathrm{AB}$ stacked bi- and multilayer graphene, at frequencies corresponding to the interlayer coupling $(\hbar \omega \sim$ $0.4 \mathrm{eV}=0.15 t$ ) one has an additional resonance in the conductivity (up to $\sigma / \sigma_{0} \sim 5$ ) due to the additional interband transitions. . $^{21,44-47}$ Nonetheless, this feature is significantly softened in the multilayer/graphite situation ${ }^{21}$ and, in addition, appears at frequencies much above the region where the polarizing effect discussed here is most efficient $(\hbar \omega \lesssim 0.02 t$, from Fig. 5). Our predicted dichroic behavior should not be affected by this $\mathrm{AB}$ stacking feature under these conditions. Moreover, this stacking-induced resonance seems much weaker than the intraband peaks that we focus on (according to Fig. 7, $\sigma / \sigma_{0} \gtrsim 100$ ), and should be absent altogether if there is no perfect stacking.

${ }^{21}$ A. B. Kuzmenko, E. van Heumen, F. Carbone, and D. van der Marel, Phys. Rev. Lett. 100, 117401 (2008).

${ }^{22}$ K. F. Mak, M. Y. Sfeir, J. A. Misewich, and T. F. Heinz, Proc. Natl. Acad. Sci. USA 107, 14999 (2010).

${ }^{23}$ J. D. Jackson, Classical Electrodynamics (John Wiley \& Sons Inc, New York, 1998).

${ }^{24}$ For a rectangular cavity, the modes $\mathrm{TE}_{10}$ and $\mathrm{TE}_{01}$ are linearly polarized along the two directions $(a, b)$. For that reason, we need a linear superposition of the two to generate an arbitrary linear polarization state. The simplest situation is a square waveguide, where the two modes are degenerate, and have the same frequencydependent impedance.

${ }^{25}$ J. Bai, X. Duan, and Y. Huang, Nano Lett. 9, 2083 (2009).

${ }^{26}$ D. V. Kosynkin, A. L. Higginbotham, A. Sinitskii, J. R. Lomeda, A. Dimiev, B. K. Price, and J. M. Tour, Nature (London) 458, 872 (2009).

${ }^{27}$ L. Jiao, X. Wang, G. Diankov, H. Wang, and H. Dai, Nat. Nanotechnol. 5, 321 (2010).

${ }^{28}$ C. Tao, L. Jiao, O. V. Yazyev, Y.-C. Chen, J. Feng, X. Zhang, R. B. Capaz, J. M. Tour, A. Zettl, S. G. Louie et al., Nat. Phys., AOP (2011).

${ }^{29}$ X. Li, X. Tu, S. Zaric, K. Welsher, W. S. Seo, W. Zhao, and H. Dai, J. Am. Chem. Soc. 129, 15770 (2007).

${ }^{30}$ V. M. Pereira and A. H. Castro Neto, Phys. Rev. Lett. 103, 046801 (2009).

${ }^{31}$ Y.-W. Son, M. L. Cohen, and S. G. Louie, Nature (London) 444, 347 (2006).

${ }^{32}$ C. Stampfer, J. Güttinger, S. Hellmüller, F. Molitor, K. Ensslin, and T. Ihn, Phys. Rev. Lett. 102, 056403 (2009).

${ }^{33}$ P. Gallagher, K. Todd, and D. Goldhaber-Gordon, Phys. Rev. B 81, 115409 (2010)

${ }^{34}$ E. R. Mucciolo, A. H. C. Neto, and C. H. Lewenkopf, Phys. Rev. B 79, 075407 (2009).

${ }^{35}$ J. Martin, N. Akerman, G. Ulbricht, T. Lohmann, J. H. Smet, K. von Klitzing, and A. Yacoby, Nat. Phys. 4, 144 (2008). 
${ }^{36}$ J. Moser, A. Barreiro, and A. Bachtold, Appl. Phys. Lett. 91, 163513 (2007).

${ }^{37}$ A. S. Mayorov, R. V. Gorbachev, S. V. Morozov, L. Britnell, R. Jalil, L. A. Ponomarenko, P. Blake, K. S. Novoselov, K. Watanabe, T. Taniguchi et al., Nano Lett. 11, 2396 (2011).

${ }^{38}$ F. Sols, F. Guinea, and A. H. C. Neto, Phys. Rev. Lett. 99, 166803 (2007).

${ }^{39}$ J. Horng, C.-F. Chen, B. Geng, C. Girit, Y. Zhang, Z. Hao, H. A. Bechtel, M. Martin, A. Zettl, M. F. Crommie et al., Phys. Rev. B 83, 165113 (2011).

${ }^{40}$ J. A. Porto, F. J. García-Vidal, and J. B. Pendry, Phys. Rev. Lett. 83, 2845 (1999).

${ }^{41}$ Y. V. Bludov, M. I. Vasilevskiy, and N. M. R. Peres, Europhys. Lett. 92, 68001 (2010).
${ }^{42}$ A. Pachoud, M. Jaiswal, P. K. Ang, K. P. Loh, and B. Özyilmaz, Europhys. Lett. 92, 27001 (2010).

${ }^{43}$ D. K. Efetov and P. Kim, Phys. Rev. Lett. 105, 256805 (2010).

${ }^{44}$ L. M. Zhang, Z. Q. Li, D. N. Basov, M. M. Fogler, Z. Hao, and M. C. Martin, Phys. Rev. B 78, 235408 (2008).

${ }^{45}$ A. B. Kuzmenko, E. van Heumen, D. van der Marel, P. Lerch, P. Blake, K. S. Novoselov, and A. K. Geim, Phys. Rev. B 79, 115441 (2009).

${ }^{46}$ K. F. Mak, C. H. Lui, J. Shan, and T. F. Heinz, Phys. Rev. Lett. 102, 256405 (2009).

${ }^{47}$ Z. Q. Li, E. A. Henriksen, Z. Jiang, Z. Hao, M. C. Martin, P. Kim, H. L. Stormer, and D. N. Basov, Phys. Rev. Lett. 102, 037403 (2009). 